Trinity University

Digital Commons @ Trinity

Psychology Faculty Research

Psychology Department

2-2009

\title{
Training Forgetting of Negative Material in Depression
}

Jutta Joormann

Paula T. Hertel

Trinity University, phertel@trinity.edu

J. LeMoult

Ian Henry Gotlib

Follow this and additional works at: https://digitalcommons.trinity.edu/psych_faculty

Part of the Psychology Commons

Publication Details

Journal of Abnormal Psychology

\section{Repository Citation}

Joormann, J., Hertel, P.T., LeMoult, J., \& Gotlib, I.H. (2009). Training forgetting of negative material in depression. Journal of Abnormal Psychology, 118(1), 34-43. doi: 10.1037/a0013794

This Article is brought to you for free and open access by the Psychology Department at Digital Commons @ Trinity. It has been accepted for inclusion in Psychology Faculty Research by an authorized administrator of Digital Commons@ Trinity. For more information, please contact jcostanz@trinity.edu. 


\title{
Training Forgetting of Negative Material in Depression
}

\author{
Jutta Joormann \\ University of Miami \\ Joelle LeMoult \\ University of Miami
}

\author{
Paula T. Hertel \\ Trinity University \\ Ian H. Gotlib \\ Stanford University
}

\begin{abstract}
In this study, the authors investigated whether training participants to use cognitive strategies can aid forgetting in depression. Participants diagnosed with major depressive disorder (MDD) and neverdepressed participants learned to associate neutral cue words with a positive or negative target word and were then instructed not to think about the negative targets when shown their cues. The authors compared 3 different conditions: an unaided condition, a positive-substitute condition, and a negative-substitute condition. In the substitute conditions, participants were instructed to use new targets to keep from thinking about the original targets. After the training phase, participants were instructed to recall all targets when presented with the cues. MDD participants, in contrast with control participants, did not exhibit forgetting of negative words in the unaided condition. In both the negative and positive substitute conditions, however, MDD participants showed successful forgetting of negative words and a clear practice effect. In contrast, negative substitute words did not aid forgetting by the control participants. These findings suggest that training depressed individuals to use cognitive strategies can increase forgetting of negative words.
\end{abstract}

Keywords: depression, memory, cognition, emotion, attention

Memories affect emotion regulation in important ways. Investigators have demonstrated that memories of unpleasant events fade faster than do memories of pleasant events and that this differential fading is associated with happiness (Walker, Skowronski, \& Thompson, 2003). Researchers have also found that recalling positive autobiographical memories can repair an induced negative mood state (Joormann \& Siemer, 2004) and that remembering positive events and forgetting negative events is associated with increased well-being over the lifespan (Charles, Mather, \& Carstensen, 2003). Although the strong association between memory and emotion usually works in our favor, enhanced memory for traumatic events and the role of biased recall in maintaining depression demonstrate that it can come at a cost. Preferential recall of negative compared with positive material is one of the most robust findings in the depression literature (Matt, Vazquez, \& Campbell, 1992). Recent reviews suggest that depressed people demonstrate increased elaboration of negative material and tend to respond to negative life events and mood states with rumination (e.g., Mathews \& MacLeod, 2005). Rumination makes negative memories more accessible and more likely to be easily retrieved, resulting in a vicious circle of rumination, mood-congruent recall,

Jutta Joormann and Joelle LeMoult, Department of Psychology, University of Miami; Paula T. Hertel, Department of Psychology, Trinity University; Ian H. Gotlib, Department of Psychology, Stanford University.

This research was supported by National Institute of Mental Health Grant MH59259 to Ian H. Gotlib.

Correspondence concerning this article should be addressed to Jutta Joormann, Department of Psychology, University of Miami, Coral Gables, FL 33124. E-mail: jjoormann@psy.miami.edu and sustained negative affect (Lyubomirsky, Caldwell, \& NolenHoeksema, 1998). Indeed, the frequent experience of unintentional and often uncontrollable negative thoughts and memories has been related to both the maintenance of depressive episodes and the likelihood of recurrence (Nolen-Hoeksema, 2000; NolenHoeksema \& Larson, 1999; Roberts, Gilboa, \& Gotlib, 1998). In this context, therefore, the ability to forget negative material may play an important role in emotion regulation in general and, more specifically, may also provide insight into processes that underlie the onset and maintenance of depressive disorders. The goal of this study was to investigate whether training depressed participants in the use of specific cognitive strategies to suppress the retrieval of negative words could aid their forgetting.

Recent work by Mathews and others provided the first evidence that cognitive biases play a causal role in maintaining emotional disorders and that training sessions designed to modify these biases reduce stress reactivity and state anxiety in high-traitanxious participants (e.g., MacLeod, Rutherford, Campbell, Ebsworthy, \& Holker, 2002; Mathews \& Mackintosh, 2000). Previous studies, however, have used cognitive bias manipulations to change attention to valenced stimuli or interpretation of ambiguous stimuli; these manipulations are more relevant to anxiety disorders than they are to depression (see Mathews \& MacLeod, 2005). Given the strength of the associations among remembering, rumination, and depression, training depressed participants to suppress and forget negative material seems a particularly promising approach to the development of effective methods of remediation for this disorder. Indeed, in contrast with nondepressed persons, depressed individuals typically make little effort to suppress negative thoughts (Lyubomirsky \& Nolen-Hoeksema, 1993). 
Recent studies on intentional forgetting suggest that training in the suppression of unwanted thoughts can have enduring consequences for subsequent recall. In a series of studies, Anderson and Green (2001) demonstrated that people are able to forget unwanted material if they actively prevent the retrieval of this material during a suppression training phase. Anderson and Green's 'think/ no-think" (TNT) procedure requires participants to practice suppressing target words when faced with multiple occurrences of the cues that they had learned to associate with them. The authors found that when people consistently prevent memories from entering awareness, the subsequent deliberate recall of suppressed targets becomes increasingly difficult. Participants in these studies first learned unrelated word pairs to criterion. In the next phase (TNT phase), the first members of some pairs were presented as cues for recalling the second member (respond condition), and the first members of other pairs served as cues to stop the second member from coming to mind (suppress condition). Finally, after a varied number of trials in which participants made responses to some cues and suppressed responses to others, participants were asked to recall all targets. Using this TNT design, Anderson and Green showed that the level of recalling previously suppressed targets dropped below the baseline recall of targets not cued in the second phase. Moreover, the greater the number of suppression trials, the fewer the targets recalled on the final test. Therefore, it appears that keeping words from coming to mind during the training phase makes the subsequent recall of these thoughts or memories more difficult. These findings indicate that suppression training might be a useful tool to counteract biased recall in depressive disorders.

Anderson and Green's (2001) findings of below-baseline forgetting have been replicated (e.g., Anderson et al., 2004; Joormann, Hertel, Brozovich, \& Gotlib, 2005), although there have also been both nonreplications (e.g., Bulevich, Roediger, Balota, \& Butler, 2006) and studies with inconsistent findings of belowbaseline suppression (Hertel \& Calcaterra, 2005; Hertel \& Gerstle, 2003). It is important to note that Hertel and Calcaterra (2005) provided evidence that final recall is influenced by the use of strategies during the TNT phase. In their study, Hertel and Calcaterra compared recall following aided and unaided TNT training. In the aided condition, participants were provided with substitute words and the instruction to use these substitutes during the TNT phase to prevent the original target from coming to mind. On the final recall test, participants in the aided condition demonstrated stronger forgetting than did participants in the unaided condition.

The experiments by Anderson and Green (2001) and Hertel and Calcaterra (2005) demonstrated effects of deliberate thought suppression on later attempts to remember. It is important to note, however, that these studies were conducted with nonclinical samples and with nonemotional material. Addressing this issue, recent studies have begun to study intentional forgetting of emotional material using this task (e.g., Depue, Banich, \& Curran, 2006; Depue, Curran, \& Banich, 2007). Depue et al. (2006), for example, paired neutral faces that served as cues with neutral and negative words (Experiment 1) or with neutral and negative pictures (Experiment 2). In the final test phase, participants exhibited belowbaseline forgetting for both negative and neutral items in a recognition task. It is interesting that participants forgot significantly more negative than neutral material in the no-think condition. Hertel and Gerstle (2003) used a variant of Anderson and Green's
TNT paradigm to investigate forgetting of emotional material in dysphoric and nondysphoric students. Participants learned to associate initially neutral nouns with positive or negative adjectives that served as cues (e.g., gloomy cottage vs. splendid cottage; slashed skin vs. smooth skin). During the learning phase, participants were instructed to form a self-referential mental image for each pair. Hertel and Gerstle found that, on the final test of cued recall, dysphoric students recalled more previously suppressed nouns than did controls, regardless of the valence of the cue. To further examine forgetting of emotional material in a diagnosed sample, Joormann et al. (2005) employed the TNT paradigm with clinically depressed participants. Participants learned to associate neutral nouns with either positive or negative targets. In the suppression or TNT phase, participants were provided with multiple occasions to suppress either positive or negative targets. In the final recall test, depressed participants successfully forgot negative targets and recalled fewer negative targets as more opportunities for suppression had been provided.

These findings provide first evidence that training depressed participants in intentional forgetting could prove to be an effective strategy to counteract the consistently reported enhanced recall of negative material in depression. The typical suppression training during the TNT phase, however, does not provide participants with any guidance of how to keep unwanted material from coming to mind. Consequently, thought suppression in this task requires considerable cognitive control. Hertel and Calcaterra (2005) demonstrated that intentional forgetting can be strengthened when specific strategies are provided that help to constrain the focus of attention during attempts to suppress in the TNT phase. Providing such strategies may be particularly important when training intentional forgetting in depressed participants. Indeed, depression has been found to be characterized by deficits in cognitive control and in the initiation of strategies in otherwise unstructured tasks (see Hertel, 2000). For example, in a series of studies, Hertel and collaborators demonstrated that providing depressed participants with specific instructions and strategies that focus attention on the task at hand can alleviate depressive deficits in performance on memory tasks. Therefore, we propose that depressed participants will profit from receiving training in the use of specific strategies that aid forgetting. In the current study, we investigated whether forgetting in depressed participants can be strengthened through training in the use of specific strategies for suppressing negative material during the TNT phase.

In addition to comparing an aided and an unaided condition, we also examined the effectiveness of different suppression strategies. Previous research suggests that dysphoric participants often use negative material to distract themselves from negative thoughts and memories (Wenzlaff, Wegner, \& Roper, 1988). Because of the high accessibility of negative concepts in depression, the distracting material that is most available is likely to be negative (Dalgleish \& Yiend, 2006). Wenzlaff et al. (1988) suggested, therefore, that these attempts at distracting oneself from unwanted thoughts and memories are likely to be counterproductive in depression. To test this proposition in the present study, we compared the effectiveness of using positive and negative thought substitutes in the suppression training phase in fostering forgetting of negative material.

Given the proposed depression-associated deficit in cognitive control, we predicted that depressed participants would profit considerably from the use of cognitive strategies and, therefore, 
would exhibit greater forgetting of negative material in the aided than in the unaided conditions. In addition, following Wenzlaff et al. (1988) and Dalgleish and Yiend (2006), we investigated whether the use of positive substitute words would result in increased forgetting, whereas the use of negative substitute words would be ineffective or even counterproductive.

\section{Method}

\section{Overview}

The procedure consisted of four different parts. During the learning phase, participants were asked to memorize weakly related noun pairs, each consisting of an emotionally neutral cue and a negative or positive target (e.g., mushroom-hostage; curtainhumor). Participants were required to reach the criterion of $50 \%$ correctly recalled targets on subsequent learning tests to move on to the TNT phase. In the TNT phase, cues were presented either 2 or 12 times, and participants practiced responding with positive targets to cues presented in green font and suppressing negative targets to cues presented in red font. Suppression was practiced under one of three conditions: unaided suppression, suppression aided by positive substitutes, or suppression aided by negative substitutes. All substitutes were related to their corresponding cues (e.g., mushroom-poison). Finally, in the recall phase, participants were asked to recall all original targets associated with the cues, regardless of whether they had practiced recalling or suppressing them during training. In a second test of cued recall, we provided the first letter of the target in addition to the cue to coax retrieval. Participants in the substitute conditions were also asked to recall the substitutes.

\section{Participants and Design}

Procedure for selection. Participants were recruited from advertisements posted on a frequently visited Internet page covering the San Francisco, California, area. Respondents took part in a phone interview, during which initial screening criteria were assessed (e.g., between 18 and 60 years old, no history of severe head trauma, no learning disability, and no color blindness). Those who were potentially eligible were invited to come into the laboratory and were administered the complete Structured Clinical Interview for the Diagnostic and Statistical Manual of Mental Disorders (4th ed.; SCID; First, Spitzer, Gibbon, \& Williams, 1997) by a trained interviewer to assess current and lifetime diagnosis.

A total of 75 currently depressed participants and 62 neverdepressed control participants took part in the learning phase. Of those participants, 26 depressed and 17 controls did not meet the criterion on the initial learning tests and did not continue. In addition, we randomly excluded 4 depressed participants to maintain equal cell sizes.

Final sample and design. Our final sample consisted of 45 currently depressed participants and 45 controls. The depressed group consisted of individuals who, at the time of testing, met Diagnostic and Statistical Manual of Mental Disorders (4th ed.; American Psychiatric Association, 1994) criteria for major depressive disorder (MDD) but not for psychotic symptoms, bipolar disorder, and alcohol or substance abuse within the past 6 months.
Five of the MDD participants were also diagnosed with dysthymia, 5 with anxiety disorders, and 2 with eating disorders. The nondepressed control group consisted of individuals with no current diagnosis and no history of any Axis I disorder. Participants in the depressed group were slightly but significantly older than participants in the nondepressed control group (depressed group, $M=$ $36.6, S D=11.7$; control group, $M=31.5, S D=10.7)$, $t(88)=$ $2.2, p<.03$, but did not differ in years of education completed (depressed group, $M=15.0, S D=3.5$; control group, $M=14.9$, $S D=4.4), t(88)<1$, or proportion female (.69 and .78 in the depressed and control groups, respectively), $\chi^{2}(1, N=90)<1$.

Participants were randomly assigned to suppression conditions (unaided, positive substitutes, or negative substitutes) and the three materials conditions (used for counterbalancing; see below), subject to the constraint of equal cell sizes of 5 . At the end of the session, participants completed the Beck Depression Inventory (BDI-II; Beck, Steer, \& Brown, 1996), a reliable and widely used self-report measure of depressive symptoms. The BDI-II consists of 21 items that assess the level of depressive symptoms present in the past 2 weeks. The mean score on the BDI-II was 28.38 ( $S D=$ $11.32)$ in the depressed group and $1.76(S D=1.96)$ in the control group, $t(88)=15.54, p<.001$. BDI-II scores did not differ as a function of assigned suppression condition within either group (within the depressed group, $M=29.9, S D=13.40 ; M=30.1$, $S D=8.63$; and $M=25.2, S D=11.52$ for the unaided, positive substitute, and negative substitute groups, respectively), $F(2$, 42 ) $<1$, (within the control group, $M=2.4, S D=2.02 ; M=$ $1.4, S D=1.88$; and $M=1.5, S D=1.92$; for the unaided, positive substitute, and negative substitute groups, respectively), $F(2,42)=1.24, p>.05$.

\section{Materials}

Word pairs. Cues and targets were selected using the Affective Norms for English Words (Bradley \& Lang, 1999). Nouns with valence ratings below 4.0 were eligible for the negative category, between 4.0 and 6.0 for the neutral category and above 6.0 for the positive category. Eighteen negative, 18 positive, and 36 neutral nouns were selected (mean valence ratings of 2.3, 7.7, and 5.5, respectively). Word selection was also based on average word length, frequency, and arousal. The final lists of negative and positive words did not differ significantly in word length (negative $M=5.9, S D=1.4$; neutral $M=5.6, S D=1.5$; positive $M=6.1$, $S D=1.7$ ), word frequency (negative $M=13.7, S D=20.8$; neutral $M=30.1, S D=41.4$; positive $M=20.2, S D=20.7$ ), or arousal (negative $M=5.6, S D=0.8$; neutral $M=4.1, S D=0.6$; positive $M=5.6, S D=1.0$ ), all $t \mathrm{~s}(34)<1$. In addition, we used the University of South Florida association norms to limit the number of forward and backward associations within the positive and negative lists and between the neutral and emotional word lists (Nelson, McEvoy, \& Schreiber, 1998).

Within each valence, the words were organized into sets containing six words each, and these sets were balanced on mean word length, valence, arousal, and concreteness (all $p$ s $>.05$ ). Each of the six sets of neutral words was paired with a set of negative or positive words. Within these paired sets, individual words were paired by choosing those for which no reported forward or back- 
ward associations were found (on the basis of the University of South Florida association norms; Nelson et al., 1998). The three sets of positive word pairs and three sets of negative word pairs were rotated systematically across the three-cue presentation $(0,2$, or 12) so that each pair would later appear in the baseline condition for one third of the participants, as a target to be suppressed or responded to twice by another third, and as a target to be suppressed or responded to 12 times for a final third. An additional 14 neutral words were selected from the same pool, with the same criteria, and paired to serve as seven fillers in the learning and training phases.

Substitute words (18 negative and 18 positive) were also selected using Affective Norms for English Words (Bradley \& Lang, 1999) and the University of South Florida association norms (Nelson et al., 1998) on the basis of the same affective rating cutoffs $(M \mathrm{~s}=6.5$ and 2.1, respectively). In contrast with the targets, substitutes were chosen to maximize the number of forward and backward associations with their corresponding cues. We selected thought substitutes on the basis of their a priori association with the cues to increase interference from the substitutes and to quickly establish their connection with the cues during the TNT phase.

\section{Procedure}

The procedure largely replicates Joormann et al. (2005). We used Superlab Pro software (Version 2.04; Cedrus Corporation, San Pedro, CA) to run all computer tasks; words were presented in black 24-point font, centered on a white screen, unless otherwise noted. Displays were separated by a blank screen for $500 \mathrm{~ms}$. The experimental session lasted 50-120 min, and participants were paid $\$ 25$ per hour.

Learning phase. Forty-three word pairs appeared sequentially for $5 \mathrm{~s}$ each. Seven of these were filler pairs, three of which were presented at the beginning of the list, one in the middle, and three at the end. All other word pairs appeared in a randomized block order, with each block of six trials containing one pair from each of the six sets. Participants were instructed to say the word pairs aloud and try to remember them for a later test of attention.

After the participants viewed the complete list of word pairs once, a feedback test was administered. When presented with each cue word, participants attempted to recall the target aloud. Once participants replied (or when 5,200 ms elapsed), the target appeared in blue for $2 \mathrm{~s}$. Participants were instructed to use it to reinforce their knowledge of the word pair. If fewer than half of the word pairs were correctly recalled, an additional feedback test was administered with differently ordered cue words. Participants were given four chances to correctly recall at least half of the word pairs before moving on to the TNT phase. Those who failed to reach the criterion were asked to complete questionnaires and were thanked for their participation.

Presentation of substitutes (for the substitute conditions only). Participants saw a list of cues from the two sets destined for suppression practice; each cue was followed by its related substitute. Neither cues nor substitutes were provided for the set of negative pairs reserved for use in the baseline condition. Each new pair appeared for $3 \mathrm{~s}$. Having been told about the TNT phase, participants were instructed to study and say the new word pairs aloud so that they could use the substitutes to help them suppress targets in the next task.

TNT phase. We started participants with a short practice task to familiarize them with the TNT procedure. In both the practice task and the main TNT task, each trial started with a series of crosses for $200 \mathrm{~ms}$. Next, a cue appeared in either a green or a red font for $3 \mathrm{~s}$ (or less if the participant responded sooner). When the cue was green, participants were instructed to respond with the target, just as they had done in the learning phase. (The blue feedback target was displayed for $500 \mathrm{~ms}$ in the main TNT phase, only when participants responded incorrectly; during practice trials, it was presented regardless of the participants' response.) When the cue was red, participants were instructed to focus on and comprehend the cue word but to avoid saying or thinking about the associated target. Those who had learned a substitute were asked to think about and reply with the substitute word instead. If participants mistakenly responded with the original target, a series of very large red $X \mathrm{~s}$ was displayed for $500 \mathrm{~ms}$. For participants who had learned a substitute, the same series of red $X \mathrm{~s}$ was displayed if they responded with an incorrect substitute word or not at all. Regardless of participants' accuracy in recalling the substitute, it appeared in blue font for $500 \mathrm{~ms}$ at the end of every suppression trial.

During the practice task, six filler cue words appeared in green once or twice each and one filler cue word appeared in red eight times. Participants in the substitute conditions were given a substitute to practice thinking about when red cues appeared. At the end of the practice task, participants completed a short form to ensure that they understood and complied with the instructions.

The main task consisted of 244 trials. Six green cues for positive targets and six red cues for negative targets were each presented twice; similarly, six cues for positive and six for negative targets were each presented 12 times. In addition, six green cues for neutral filler targets were presented 12 times; two filler cues occurred at the beginning and two at the end of the list. The first presentation of the twice-presented cue words occurred in the first half of the trials. All cue words that were to be presented 12 times were displayed before the next random iteration began.

Final recall tests. The final recall phase had three components. In the cued recall task, each cue appeared for $4 \mathrm{~s}$, or less if the participant responded sooner. Participants were instructed to recall and say aloud all original targets in response to the presented cues, regardless of whether they had suppressed or responded with them in the training phase. Four filler words were followed by six randomized blocks of one cue from each of the six sets. Cues appeared in a randomized block order that was the same for all participants; each block contained a cue from each of the six sets of pairs. If the substitute word was also remembered, participants were instructed to say that word aloud and, if possible, specify which was the substitute and which the original response. After the cued recall task, the same test occurred a second time but with cues that were followed by the first letter of the original target (stemcued recall). Participants were told to use the letter to help them recall the target. Finally, participants in the substitute conditions were given a sheet of paper that listed all cue words that had been paired with positive or negative substitutes and were asked to write down the substitute words. 


\section{Results}

Each recall measure was submitted to an analysis of variance (ANOVA) with between-subjects factors for group (MDD, control) and suppression condition (unaided, positive substitutes, negative substitutes). Most analyses also included within-subject factors for the number of cue presentations $(0,2,12)$ and instruction (respond, suppress) during the TNT phase. Some of the cue members of the originally learned pairs (the cues in the 0 condition) were never presented during the TNT phase. The recall of these targets on the final test served as the baseline condition. We also included a between-subjects factor for the materials condition (3 levels) in each analysis to reduce error variance; we do not report effects involving that factor. The dependent variables were the percentages of recalled targets (from a total of six items per condition). The significance level was set at .05 . We do not report significant effects that were qualified by higher order interactions. Other unreported effects in the overall designs were all nonsignificant; for these effects, the lowest $p$ value was .10 .

\section{Cued Recall}

Our predictions concerned the percentage of targets recalled on the first test following the TNT phase. The first ANOVA conducted on this measure included within-subjects factors for instruction (respond with positive, suppress negative targets) and the number of cue presentations $(0,2,12)$ during the suppression training phase. First, as is always the case in TNT experiments, the interaction of instruction (respond, suppress) with the number of cue presentations $(0,2,12)$ was significant, $F(2,144)=78.10$, $M S E=218.61, p<.001, \eta_{p}^{2}=.52$. Thus, the number of words that participants recalled in the respond versus suppress conditions depended on the number of times the cue words were presented. Second, the effect of instruction depended on the suppression conditions (unaided, positive substitutes, negative substitutes), $F(2,72)=8.43, M S E=376.02, p=.001, \eta_{p}^{2}=.19$. Third, the three-way interaction of group, suppression condition, and the number of cue presentations was significant, $F(4,144)=4.34$, $M S E=191.87, p=.002, \eta_{p}^{2}=.11$. To understand these interactions and to evaluate specific predictions, we conducted further analyses separately within the respond and suppress conditions.

In the respond condition, recall of positively valenced targets depended significantly on how many times their cues were presented during the TNT phase, $F(2,144)=80.78, M S E=148.40$, $p<.001, \eta_{p}^{2}=.53(M \mathrm{~s}=76,91$, and 99 for 0,2 , and 12 presentations, respectively). In addition, MDD participants recalled fewer positive targets than did controls $(M=86$ vs. 90$)$, but the difference was only marginally significant, $F(1,72)=3.78$, $M S E=278.80, p=.056, \eta_{p}^{2}=.05$.

Our predictions all pertained to the percentage of recalled targets following training in the suppression of negative words. In this analysis, we used the linear trend across the number of cue presentations $(0,2,12)$ as the within-subjects factor to evaluate below-baseline forgetting. The three-way interaction of group, suppression condition, and this linear trend was significant, $F(2$, $72)=4.76, M S E=266.97, p=.011, \eta_{p}^{2}=.12 .{ }^{1}$ Figure 1 depicts the means for the control group, and Figure 2 depicts the means for the MDD group. Follow-up tests were conducted within each group.

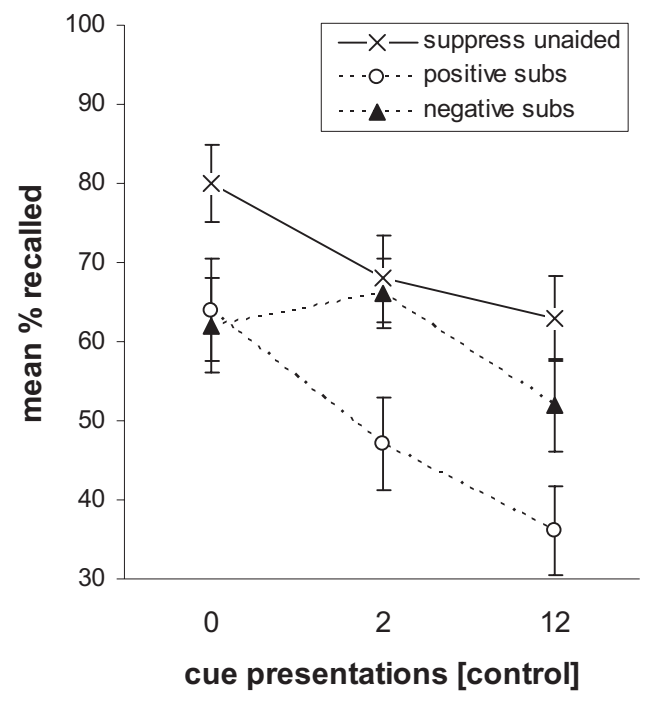

Figure 1. Mean percentage of targets recalled by the control participants after suppression training as a function of condition (suppress unaided, positive subs, and negative subs) and number of cue presentations. Subs $=$ substitutes. Error bars $=1 S E$.

As seen in Figure 1, forgetting in the control group depended upon the condition for suppression, $F(2,36)=6.62, M S E=$ 784.96, $p=.004, \eta_{p}^{2}=.27$. Tukey's honestly significant difference revealed that control participants who were trained to use positive substitutes recalled fewer targets than did participants in the other conditions, which did not differ significantly. Across conditions, control participants showed evidence of belowbaseline forgetting, $F(1,36)=27.62, M S E=279.33, p<.001$, $\eta_{p}^{2}=.43$. Within conditions, control participants exhibited belowbaseline forgetting in the unaided condition, $F(1,12)=12.50$, $M S E=2083.00, p<.01, \eta_{p}^{2}=.51$, and in the positive substitutes condition, $F(1,12)=48.28, M S E=6258.87, p<.001, \eta_{p}^{2}=.80$. In the negative substitutes condition, a significant quadratic trend was obtained, $F(1,12)=4.84, \operatorname{MSE}=750.10, p<.001, \eta_{p}^{2}=.29$. As seen in Figure 1, recall differed significantly only between the condition in which the cue was presented twice and the condition in which the cue was presented 12 times, $t(14)=2.26, p<.05$. Thus, control participants exhibited training effects in the forgetting of negative targets in the unaided and the aided conditions. Training in the use of positive thought substitutes, however, further aided forgetting of negative targets in this group.

As seen in Figure 2, the interaction of suppression condition (unaided, positive substitutes, negative substitutes) with the linear trend across the number of cue presentations was significant for MDD participants, $F(2,36)=8.23, M S E=254.62, p=.001, \eta_{p}^{2}=$ .31. Follow-up tests examined means within each suppression condition. MDD participants showed no evidence of below-baseline forgetting in the unaided condition, $F(1,12)=1.14, p>.05$, but exhibited below-baseline forgetting when using both positive substitutes, $F(1,12)=15.36, M S E=3342.87, p=.02, \eta_{p}^{2}=.56$, and

\footnotetext{
${ }^{1}$ These results did not change when age of the participants or number of correctly recalled words on the final learning trial were included as covariates.
} 


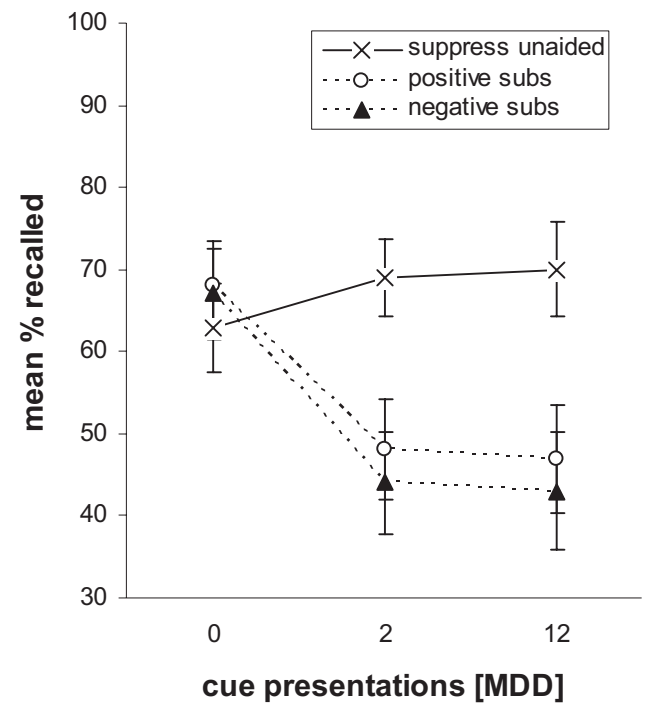

Figure 2. Mean percentage of targets recalled by the depressed participants after suppression training as a function of condition (suppress unaided, positive subs, and negative subs) and number of cue presentations. Subs = substitutes; MDD = major depressive disorder. Error bars $=1 S E$.

negative substitutes, $F(1,12)=16.04, M S E=4083.41, p<.01$, $\eta_{p}^{2}=.57$. Thus, in this group, below-baseline forgetting occurred only when substitutes were provided. Forgetting did not depend on the valence of the substitutes.

We also examined differences involving groups within each suppression condition. A significant interaction of group and cue presentation emerged only in the unaided condition, $F(1,24)=$ $8.91, M S E=2041.67, p=.006, \eta_{p}^{2}=.27$. In both the positive and negative substitute conditions, main effects in linear trends of cue presentation were the only significant findings; for positive substitutes, $F(1,24)=53.99, M S E=9375.00, p<.001, \eta_{p}^{2}=.69$; for negative substitutes, $F(1,24)=10.47, M S E=4166.88, p=$ $.004, \eta_{p}^{2}=.30$. Thus, group differences in below-baseline forgetting were obtained only in the unaided conditions.

\section{Stem-Cued Recall}

To evaluate the robustness of the forgetting of negative targets after suppression training, we conducted a second recall test by an additional cue in the form of the first letter of the target word (see Table 1). Our intent was to coax retrieval of the negative targets. The analysis of these results, restricted to negative targets, revealed the same pattern of results as that obtained in the first test. The three-way interaction of group, suppression condition, and the linear trend across the number of cue presentations was significant, $F(2,72)=3.35, M S E=287.81, p=.041, \eta_{p}^{2}=.08$. Follow-up tests yielded results similar to those obtained on the first recall test. Control participants exhibited below-baseline forgetting in the unaided condition, $F(1,12)=6.40, M S E=1333.20, p=.026, \eta_{p}^{2}=$ .35 , and when they used positive substitutes, $F(1,12)=32.06$, $M S E=4898.57, p<.001, \eta_{p}^{2}=.73$, but not when they used negative substitutes, $F(1,12)<1$. For the MDD group, no belowbaseline forgetting was found in the unaided condition, $F(1,12)<$ 1. In both the positive and the negative substitutes conditions, however, significant below-baseline forgetting emerged, $F(1$, 12) $=7.92, M S E=2676.24, p=.016, \eta_{p}^{2}=.40$, for positive substitutes; $F(1,12)=8.03, M S E=2675.80, p=.015, \eta_{p}^{2}=.40$, for negative substitutes.

In a further analysis, we compared performance across the two types of recall tests by including a within-subjects factor for test (first vs. second). The only significant outcome involving this test factor was its main effect, $F(1,72)=61.13, M S E=125.24, p<$ $.001, \eta_{p}^{2}=.46$. On average, $59 \%$ of targets were recalled on the first test and $66 \%$ on the stem-cued test.

\section{Substitutes}

The third test asked participants to recall each substitute when presented with the appropriate cue. An ANOVA was conducted with a within-subjects factor for the number of cue presentations during the TNT phase ( 2 or 12). The only significant effect in the overall design was the main effect of the number of cue presentations, $F(1,48)=21.01, M S E=185.18, p<.001, \eta_{p}^{2}=.30$. Participants recalled an average of $80 \%$ of substitutes used twice and $91 \%$ of substitutes used 12 times.

Table 1

Mean Percentage (and Standard Deviations) of Targets Recalled in the Stem-Cued Recall Task After Suppression Training

\begin{tabular}{cccccccc}
\hline & \multicolumn{3}{c}{ Control group } & & \multicolumn{3}{c}{ MDD group } \\
\cline { 2 - 3 } \cline { 7 - 7 } Condition & Unaided & Pos subs & Neg subs & & Unaided & Pos subs & Neg subs \\
\hline Respond & & & & & & & \\
0 & $84.44(16.01)$ & $78.88(18.32)$ & $85.55(13.89)$ & & $75.56(21.70)$ & $76.66(18.69)$ & $79.99(20.11)$ \\
2 & $98.88(4.30)$ & $87.77(16.02)$ & $96.66(6.90)$ & & $93.33(8.45)$ & $91.11(12.38)$ & $91.11(18.76)$ \\
12 & 100 & $96.67(9.34)$ & $96.67(6.90)$ & & $97.78(5.87)$ & $93.33(10.54)$ & 100 \\
Suppress & & & & & & & \\
0 & $84.44(17.21)$ & $72.22(20.58)$ & $71.11(20.38)$ & & $68.88(19.79)$ & $73.33(18.69)$ & $72.22(23.28)$ \\
2 & $73.33(16.43)$ & $55.56(22.41)$ & $70.00(20.11)$ & & $72.22(14.99)$ & $55.56(27.93)$ & $58.89(22.60)$ \\
12 & $71.11(14.73)$ & $46.66(26.13)$ & $67.78(20.38)$ & $71.11(23.96)$ & $54.44(23.96)$ & $53.34(21.08)$ \\
\hline
\end{tabular}

Note. $\quad N=90$; Respond $=$ participants were asked to respond with the target during suppression training. Suppress $=$ participants were asked to suppress the target during suppression training. $0=$ baseline, $2=$ two cue presentations, $12=12$ cue presentations. Unaided = suppress unaided; pos subs = positive substitutes, neg subs $=$ negative substitutes. 


\section{Discussion}

Depression is characterized by the frequent occurrence of unintentional and often uncontrollable negative thoughts and memories. Although we are gaining a better understanding of the role that these biases play in maintaining depression, we know little about effective methods to ameliorate them. In this study, we examined whether we could train depressed individuals to forget negative material. We explicitly instructed depressed and nondepressed participants not to think about negative target words that they had learned to associate with a neutral cue word, and we gave them multiple occasions to practice suppression. We further compared an unaided suppression condition with two conditions in which we provided participants with specific cognitive strategies to aid forgetting: the use of positive and negative thought substitutes. We predicted that, given depression-associated deficits in cognitive control, training in the use of a specific strategy, such as the use of positive or negative substitutes, would aid forgetting by depressed participants. The results support our main prediction. Depressed participants benefited considerably from being provided with a strategy for forgetting negative material. Whereas only control participants exhibited below-baseline forgetting in the unaided condition, both depressed and control participants exhibited this effect when using substitutes. We also found that positive and negative substitutes were differentially effective in aiding forgetting, but only for the nondepressed participants, who could use only positive substitutes to further increase forgetting. In contrast, depressed participants did not differ in their forgetting of negative stimuli when using positive or negative substitutes. Finally, the forgetting of negative material was robust; the results remained stable even when recall was aided by the presentation of the first letter of the target in a second memory task.

In a previous study using the TNT design, we demonstrated that depressed participants profit from repeated occasions of practicing suppressing negative material and exhibit increased forgetting of negative words following suppression training (Joormann et al., 2005). In the present study, we extended these findings by demonstrating that training depressed participants in the use of specific strategies further aids forgetting. These studies, therefore, provide first evidence for the impact of cognitive training on biased processing of negative material in depression. Previous research has demonstrated that training allocation of attention away from emotional material and training the interpretation of ambiguous material toward benign interpretations in student samples or in participants scoring high on anxiety measures results in reduced post-training state anxiety and reduced reactivity when participants are exposed to a laboratory stressor. In one of the first studies to use this approach, MacLeod et al. (2002) experimentally induced differential attentional responses to emotional stimuli in college students and found that, compared with participants who were trained to focus on negative material, participants who were trained to avoid negative stimuli demonstrated a reduced tendency to respond to a subsequent stress task with increases in negative mood ratings. Similarly, Mathews and Mackintosh (2000) trained participants to interpret ambiguous information in a benign or threatening manner and found that training reduced both negative interpretation biases to novel stimuli and post-training state anxiety scores. Similar results have been reported in a number of follow-up studies, all conducted under the umbrella of cognitive- bias modification (Hirsch, Mathews, \& Clark, 2007; Wilson, MacLeod, Mathews, \& Rutherford, 2006; Yiend, Mackintosh, \& Mathews, 2005).

Modifications of cognitive biases in attention and interpretation are more relevant to our understanding of anxiety disorders than to our understanding of depression. In contrast with anxiety disorders, depression is characterized primarily by sustained processing and increased elaboration of negative material, which result in biased memory (for a review, see Mathews \& MacLeod, 2005). Consequently, cognitive-bias modification paradigms that focus on reducing biases in interpretation and attention are less likely to be effective in depression. In contrast, training intentional forgetting of negative material targets a cognitive bias that has been consistently linked to depression (Matt et al., 1992). Our findings suggest that depressed participants can learn to forget negative material if we instruct them to suppress previously learned negative material, provide them with multiple occasions to practice suppression, and provide them with strategies that constrain their attention and thereby aid suppression. Indeed, we found transfer of our suppression training to participants' performance on a memory task. In this study, we did not assess whether this training also reduced negative affect or stress reactivity in the depressed participants. In this regard, it is unclear whether the stress-reactivity tasks (e.g., unsolvable anagrams) that have been used in previous training studies in anxiety would be useful in depression (see Mathews \& MacLeod, 2005). Nevertheless, the demonstration in this study of an effect of training on biased processing in depression is an important first step in evaluating cognitive training in this disorder. Future studies are required to examine the important question of whether suppression training also affects other aspects of depressive disorders. Recent studies that have focused on training depressed and dysphoric participants to recall more specific and less overgeneral autobiographic memories have reported that this training affects emotional vulnerability (e.g., Moberly \& Watkins, 2006; Philippot, Schaefer, \& Herbette, 2003; Raes, Hermans, Williams, \& Eelen, 2006).

Our findings further elucidate when and why intentional forgetting is observed in Anderson and Green's (2001) TNT design. Our results are consistent with Hertel and Calcaterra's (2005) finding that forgetting of nonemotional material in this task is strengthened by the use of thought substitutes. In the traditional TNT phase, participants are instructed merely to avoid thinking about the target word when they see the red cue, without further instructions about how suppression might be achieved. The lack of guidance in the suppression phase leaves room for individual differences in the use of self-initiated strategies (as also suggested by Bulevich et al., 2006). Indeed, in a previous study in which we used this design to investigate forgetting of emotional material in depression, we found below-baseline forgetting of negative material in the depressed participants, even though we provided no strategy for the suppression training phase (Joormann et al., 2005). These participants, however, reported frequent use of thought substitutes in a questionnaire they were given after the final recall task. The use of thought substitution introduces retroactive interference and is similar to retrieval-induced forgetting, in which rehearsal of items that are closely related to a category cue prevents later recall of other previously learned items in the same category (Anderson \& Spellman, 1995). In contrast with the original targets, the substitute words used in the present study were selected so that they were a 
priori associated with the cue words. In retrieval-induced forgetting, practicing material that competes with unpracticed memories leads to reduced recall of the unpracticed words (Anderson \& Spellman, 1995). The increased forgetting of negative material when using thought substitutes in the present study might be due to a similar mechanism.

The to-be-suppressed targets in the TNT phase are similar to unwanted thoughts. Research examining the intentional suppression of unwanted thoughts has been guided by the notion of ironic control processes, which predicts that attempts to push away or suppress an unwanted thought increase its accessibility, particularly under high cognitive load (Wegner, 1994). There are a number of differences, however, between typical thoughtsuppression tasks and the TNT design. For example, whereas participants in thought-suppression studies are usually presented with the to-be-suppressed item once, participants in the TNT study are provided with multiple occasions to suppress. In addition, whereas the dependent variable in thought suppression tasks is usually the level of success of thought suppression as indexed by the number of intrusions, the dependent measure in the TNT task is subsequent recall of the to-be-suppressed items. In fact, investigators explicitly examining recall have found suppression to lead to poorer memory for the suppressed stimuli (Rassin, 2001; Wegner, Quillian, \& Houston, 1996). Still, thinking of something else when confronted with the cue might be a particularly powerful strategy that aids thought suppression and counteracts the ironic processes that are seen in other thought-suppression designs. Wegner, Schneider, Carter, and White (1987), for example, reported that participants who were given a specific alternative thought (e.g., a red Volkswagen) to use as a distractor during thoughtsuppression trials reported fewer intrusions. Similarly, Salkovskis and Campbell (1994) demonstrated that thought suppression was successful after an interval of engagement in a different task. Distraction has been found in a number of studies to curtail rumination, reduce recall biases, and repair negative mood states in depressed participants (Joormann \& Siemer, 2004; Joormann, Siemer, \& Gotlib, 2007; Lyubomirsky et al., 1998). Thus, an important difference between depressed and nondepressed participants may involve their ability to constrain attention in unstructured tasks like the TNT phase and to initiate the use of effective strategies like distraction or thought substitution (see Hertel, 2004).

Another interesting finding of this study concerns the differential effectiveness of positive and negative thought substitutes. Whereas both the depressed and nondepressed participants profited from using positive thought substitutes, depressed participants exhibited greater forgetting of negative material than did nondepressed controls when using negative thought substitutes. This pattern of findings suggests that using positive thought substitutes is a powerful strategy that aids forgetting of negative material in everyone; the effectiveness of using negative thought substitutes to aid forgetting of negative stimuli in depressed individuals, however, is intriguing. Wenzlaff et al. (1988) found that depressed participants are likely to distract themselves with other negatively valenced material when trying to suppress negative thoughts but also noted that this might be an ineffective strategy. Specifically, although negative cues might lead to effective thought suppression, the use of these cues might not be effective in changing mood states or might even exacerbate negative affect. Similarly, Dozois and Dobson (2001) suggested that because negative information in depression is densely interrelated, the distracting information that is most available is negative. Indeed, Dalgleish and Yiend (2006) reported that the attempts by depressed individuals to suppress memories led to speeded access to other negative, but not positive, material on a subsequent cued recall task. These investigators further suggested that the use of negative distractors is likely maladaptive in terms of breaking patterns of negative thinking. The results of the current study, however, suggest that negative and positive distracting thoughts can aid forgetting of negative material in depression. These findings suggest that distraction is a powerful tool that aids thought suppression and intentional forgetting in depression, independent of the content of the distracting thoughts. Given the increased accessibility of negative material in depressed individuals, negative thought substitutes may create increased interference, which leads to increased forgetting. Investigators might profitably examine this proposed mechanism more explicitly in future research.

Clearly, these findings represent only a first step in research on cognitive bias training in depression, and they have several limitations. For example, we did not assess the long-term effects of our training, nor did we investigate whether our training affects other depressive symptoms, like stress vulnerability or mood ratings. Similarly, we did not investigate whether forgetting generalizes to other memory tests, and we investigated forgetting of only negative material; future research should examine whether depressed and nondepressed people differ in their intentional forgetting of positive and neutral information. Our results demonstrate that the use of thought substitutes improves forgetting of negative material in depressed participants. It is noteworthy that exposing MDD participants to 12 opportunities to practice the use of thought substitutes did not enhance forgetting in this group beyond that observed with 2 practice opportunities. It is possible, therefore, that the initial learning of thought substitutes is sufficient for below-baseline forgetting to occur and that repeated exposure to the suppress cues and repeated use of the thought substitutes do not enhance this forgetting. That is, because the baseline cannot include either substitutes or TNT training, it is difficult to determine whether participants must go through the TNT training to increase forgetting or whether it is sufficient to provide them with a thought substitute. Future research might distinguish more explicitly between these two possibilities and increase our understanding of the mechanisms that underlie the forgetting effects observed in our study.

In addition, because we did not include a test of recall using independent cues, we cannot in this study examine the underlying mechanisms of increased intentional forgetting. Thus, it is unclear whether our training established cognitive inhibition of the to-beforgotten material or whether forgetting was entirely due to the interference created by alternative thoughts. As Anderson and Levy (2007) have pointed out, independent cues are required to isolate inhibition as the cause of forgetting. The main goal of this study, however, was to demonstrate that the use of a strategy can increase forgetting, and future research is needed to examine the underlying mechanisms of this effect.

These limitations also preclude strong statements about the potential effectiveness of our training for clinical intervention. Future studies should investigate whether the present results generalize to the personal information that plays a role in the main- 
tenance of depression. Although the present findings are encouraging, it is important to be cautious in drawing clinical implications from the results of experimental studies (e.g., Holmes, Moulds, \& Kavanagh, 2007). The present results suggest that forgetting of negative material in depression can be achieved. The question of whether suppressing emotional material is beneficial or detrimental in the treatment of depression or of other disorders like post-traumatic stress disorder was not addressed explicitly in our experiment (see Reynolds \& Brewin, 1999, for an interesting essay about this debate).

In closing, our findings suggest that training depressed participants to suppress negative material and, most important, providing them with a strategy for how to suppress increases intentional forgetting of negative material. Thus, training depressed individuals in intentional forgetting could prove to be an effective strategy to counteract ruminative tendencies and the consequent sustained processing of negative material. Similarly, Brewin (2006) suggested that CBT may work not by directly modifying negative information in memory but, instead, by producing changes in the relative accessibility of positive and negative representations such that positive memories involving the self win the retrieval competition over negative memories. Given that the intentional forgetting of negative material plays an important role in emotion regulation, this and other forms of cognitive training that have the potential to increase individuals' cognitive control may represent important methods of remediation.

\section{References}

American Psychiatric Association. (1994). Diagnostic and statistical manual of mental disorders (4th ed.). Washington, DC: Author.

Anderson, M. C., \& Green, C. (2001, March 15). Suppressing unwanted memories by executive control. Nature, 410, 366-369.

Anderson, M. C., \& Levy, B. J. (2007). Theoretical issues in inhibition: Insights from research on human memory. In D. Gorfein \& C. MacLeod (Eds.), Inhibition in Cognition (pp. 81-102). Washington, DC: American Psychological Association.

Anderson, M. C., Ochsner, K. N., Kuhl, B., Cooper, J., Robertson, E., Gabrieli, S. W., et al. (2004, January 9). Neural systems underlying the suppression of unwanted memories. Science, 303, 232-235.

Anderson, M. C., \& Spellman, B. A. (1995). On the status of inhibitory mechanisms in cognition: Memory retrieval as a model case. Psychological Review, 102, 68-100.

Beck, A. T., Steer, R. A., \& Brown, G. K. (1996). Manual for the Beck Depression Inventory-II. San Antonio, TX: Psychological Corporation.

Bradley, M. M., \& Lang, P. J. (1999). Affective Norms for English Words (ANEW): Technical manual and affective ratings. Gainesville, FL: Center for Research in Psychophysiology, University of Florida.

Brewin, C. R. (2006). Understanding cognitive behavior therapy: A retrieval competition account. Behaviour Research and Therapy, 44, 765784

Bulevich, J. B., Roediger, H. L., III, Balota, D. A., \& Butler, A. C. (2006). Failures to find suppression of episodic memories in the think/no-think paradigm. Memory and Cognition, 34, 1569-1577.

Charles, S. T., Mather, M., \& Carstensen, L. L. (2003). Aging and emotional memory: The forgettable nature of negative images for older adults. Journal of Experimental Psychology: General, 132, 310-324.

Dalgleish, T., \& Yiend, J. (2006). The effects of suppressing a negative autobiographical memory on concurrent intrusions and subsequent autobiographical recall in dysphoria. Journal of Abnormal Psychology, $115,467-473$.

Depue, B. E., Banich, M. T., \& Curran, T. (2006). Suppression of emo- tional and nonemotional content in memory. Psychological Science, 17, 441-447.

Depue, B. E., Curran, T., \& Banich, M. T. (2007, July 13). Prefrontal regions orchestrate suppression of emotional memories via a two-phase process. Science, 317, 215-219.

Dozois, D. J. A., \& Dobson, K. S. (2001). Information processing and cognitive organization in unipolar depression: Specificity and comorbidity issues. Journal of Abnormal Psychology, 110, 236-246.

First, M. B., Spitzer, R. L., Gibbon, M., \& Williams, J. B. W. (1997). Structured clinical interview for DSM-IV Axis I disorders: Clinician's version. New York: Biometrics Research Department, New York State Psychiatric Institute.

Hertel, P. T. (2000). The cognitive-initiative account of depression-related impairments in memory. In D. Medin (Ed.), The psychology of learning and motivation (Vol. 39, pp. 47-71). New York: Academic Press.

Hertel, P. T. (2004). Memory for emotional and nonemotional events in depression: A question of habit? In D. Reisberg \& P. Hertel (Eds.), Memory and emotion (pp. 186-216). New York: Oxford University Press.

Hertel, P. T., \& Calcaterra, G. (2005). Intentional forgetting benefits from thought substitution. Psychonomic Bulletin and Review, 12, 484-489.

Hertel, P. T., \& Gerstle, M. (2003). Depressive deficits in forgetting. Psychological Science, 14, 573-578.

Hirsch, C. R., Mathews, A., Clark, D. M. (2007). Inducing an interpretation bias changes self-imagery: A preliminary investigation. Behaviour Research and Therapy, 45, 2173-2181.

Holmes, E. A., Moulds, M. L., \& Kavanagh, D. (2007, December 14). Memory suppression in PTSD treatment? Science, 318, 1722.

Joormann, J., Hertel, P. T., Brozovich, F., \& Gotlib, I. H. (2005). Remembering the good, forgetting the bad: Intentional forgetting of emotional material in depression. Journal of Abnormal Psychology, 114, 640-648.

Joormann, J., \& Siemer, M. (2004). Memory accessibility, mood regulation, and dysphoria: Difficulties in repairing sad mood with happy memories? Journal of Abnormal Psychology, 113, 179-188.

Joormann, J., Siemer, M., \& Gotlib, I. H. (2007). Mood regulation in depression: Differential effects of distraction and recall of happy memories on sad mood. Journal of Abnormal Psychology, 116, 484-490.

Lyubomirsky, S., Caldwell, N. D., \& Nolen-Hoeksema, S. (1998). Effects of ruminative and distracting responses to depressed mood on retrieval of autobiographical memories. Journal of Personality and Social Psychology, 75, 166-177.

Lyubomirsky, S., \& Nolen-Hoeksema, S. (1993). Self-perpetuating properties of dysphoric rumination. Journal of Personality and Social Psychology, 65, 339-349.

MacLeod, C., Rutherford, E., Campbell, L., Ebsworthy, G., \& Holker, L. (2002). Selective attention and emotional vulnerability: Assessing the causal basis of their association through the experimental manipulation of attentional bias. Journal of Abnormal Psychology, 111, 107-123.

Mathews, A., \& Mackintosh, B. (2000). Induced emotional interpretation bias and anxiety. Journal of Abnormal Psychology, 109, 602-615.

Mathews, A., \& MacLeod, C. (2005). Cognitive vulnerability to emotional disorders. Annual Review of Clinical Psychology, 1, 167-195.

Matt, G. E., Vazquez, C., \& Campbell, W. K. (1992). Mood-congruent recall of affectively toned stimuli: A meta-analytic review. Clinical Psychology Review, 12, 227-255.

Moberly, N. J., \& Watkins, E. R. (2006). Processing mode influences the relationship between trait rumination and emotional vulnerability. Behavior Therapy, 37, 281-291.

Nelson, D. L., McEvoy, C. L., \& Schreiber, T. A. (1998). The University of South Florida word association, rhyme, and word fragment norms. Retrieved May 7, 2006, from http://www.usf.edu/FreeAssociation/

Nolen-Hoeksema, S. (2000). The role of rumination in depressive disorders and mixed anxiety/depressive symptoms. Journal of Abnormal Psychology, 109, 504-511. 
Nolen-Hoeksema, S., \& Larson, J. (1999). Coping with loss. Hillsdale, NJ: Erlbaum.

Philippot, P., Schaefer, A., \& Herbette, G. (2003). Consequences of specific processing of emotional information: Impact of general versus specific autobiographical memory priming on emotion elicitation. Emotion, 3, 270-283.

Raes, F., Hermans, D., Williams, J. M. G., \& Eelen, P. (2006). Reduced autobiographical memory specificity and affect regulation. Cognition and Emotion, 20, 402-429.

Rassin, E. (2001). Thought suppression, memory, and interrogative suggestibility. Psychology, Crime, and Law, 7, 45-55.

Reynolds, M., \& Brewin, C. R. (1999). Intrusive memories in depression and posttraumatic stress disorder. Behaviour Research and Therapy, 37, 201-215.

Roberts, J. E., Gilboa, E., \& Gotlib, I. H. (1998). Ruminative response style and vulnerability to episodes of dysphoria: Gender, neuroticism, and episode duration. Cognitive Therapy and Research, 22, 401-423.

Salkovskis, P. M., \& Campbell, P. (1994). Thought suppression induces intrusion in naturally occurring negative intrusive thoughts. Behaviour Research and Therapy, 32, 1-8.

Walker, W. R., Skowronski, J. J., \& Thompson, C. P. (2003). Life is pleasant-And memory helps to keep it that way! Review of General Psychology, 7, 203-210.
Wegner, D. M. (1994). Ironic processes of mental control. Psychological Review, 101, 34-52.

Wegner, D. M., Quillian, F., \& Houston, C. F. (1996). Memories out of order: Thought suppression and the disturbance of sequence memory. Journal of Personality and Social Psychology, 71, 680-691.

Wegner, D. M., Schneider, D. J., Carter, S. R., \& White, T. L. (1987). Paradoxical effects of thought suppression. Journal of Personality and Social Psychology, 53, 5-13.

Wenzlaff, R. M., Wegner, D. M., \& Roper, D. W. (1988). Depression and mental control: The resurgence of unwanted negative thoughts. Journal of Personality and Social Psychology, 55, 882-892.

Wilson, E. J., MacLeod, C., Mathews, A., \& Rutherford, E. M. (2006). The causal role of interpretive bias in anxiety reactivity. Journal of Abnormal Psychology, 115, 103-111.

Yiend, J., Mackintosh, B., \& Mathews, A. (2005). Enduring consequences of experimentally induced biases in interpretation. Behaviour Research and Therapy, 43, 779-797.

Received July 13, 2007

Revision received February 4, 2008

Accepted June 24, 2008 